\title{
Erratum to: A Serpent/OpenFOAM coupling for 3D burnup analysis
}

\section{Christian Castagna $^{1,3}$, Eric Cervi ${ }^{1,3}$, Stefano Lorenzi ${ }^{1,3}$, Antonio Cammi ${ }^{1,3, a}{ }^{\mathbb{D}}$,} Davide Chiesa $^{2,3}$, Monica Sisti ${ }^{2,3}$, Massimiliano Nastasi ${ }^{2,3}$, Ezio Previtali ${ }^{3}$

${ }^{1}$ Department of Energy, CeSNEF (Enrico Fermi Center for Nuclear Studies), Politecnico di Milano, Via La Masa 34, 20156 Milan, Italy

2 Department of Physics "G. Occhialini”, University of Milano-Bicocca, Piazza della Scienza 3, 20126 Milan, Italy

${ }^{3}$ INFN Section of Milano-Bicocca, Piazza della Scienza 3, 20126 Milan, Italy

Published online: 26 June 2020

() Società Italiana di Fisica and Springer-Verlag GmbH Germany, part of Springer Nature 2020

Erratum to: Eur. Phys. J. Plus (2020) 135:433

https://doi.org/10.1140/epjp/s13360-020-00427-3

During production, mistakes have been introduced.

In Table 2. "gd Radial direction" should read "Radial direction".

In Table 3, in row "Pu-239", the entries in columns 14 and 15 were wrong and have been corrected.

The original article has been corrected.

The original article can be found online at https://doi.org/10.1140/epjp/s13360-020-00427-3.

\footnotetext{
a e-mail: antonio.cammi@polimi.it (corresponding author)
} 